\title{
ADLIF: a new large-displacement beam-based flexure joint
}

\author{
X. Pei and J. Yu \\ School of Mechanical Engineering and Automation, Beihang University, Beijing 100083, China
}

Received: 28 February 2011 - Revised: 17 June 2011 - Accepted: 26 July 2011 - Published: 29 August 2011

\begin{abstract}
A flexure joint is an important component in flexure mechanisms. Most of well known flexure joints have always a trade-off among such performances as precision, stiffness, and stroke, which heavily affect the overall performances of flexure mechanisms. In this paper, a new flexure joint, named an anti-symmetric double leaf-type isosceles-trapezoidal flexure joint (ADLIF), is introduced. The joint is constructed by two leaf-type isosceles-trapezoidal flexure (LITF) building blocks in an anti-symmetrical form. In order to investigate such characteristics as precision, stiffness and stroke, two ADLIFs with different structural parameters are compared with a cartwheel hinge. In addition, a simple and accurate pseudo-rigid body (PRB) joint model of the ADLIF is formulated to simplify the parametric model and achieve the structural optimization. The results show that the ADLIF can gain a great improvement in precision as well as maintain other characteristics such as stiffness and ranges of motion similar. Even the ADLIF gets more than 16 times improvement in precision in the case that the rotational angle is less than five degrees $\left(5^{\circ}\right)$. The ADLIF can thus be used for the replacement of the cartwheel joint in some precision application fields.
\end{abstract}

\section{Introduction}

Flexure mechanisms are devices that attain motion by means of elastic deformation of flexures. They have been utilized in many applications, in particular as precise instruments (Her and Chang, 1994; Kota et al., 1999; Onillon et al., 2003; Pernette et al., 1997; Slocum, 1992) due to a number of advantages including low cost, reduced weight and smooth motion. Besides, simplified, especially monolithic manufacturing for the flexure mechanisms can cut down the number of work pieces; sequentially diminish the errors brought by assembly.

As one of the most important elements in flexure mechanisms, flexure joints transfer the motion and energy, and ensure the movement and precision that the specified applications required. One of the commonly-used flexure joints is notch hinges (Lobontiu, 2003), which provide a high precision and a large stiffness but a very limited stroke. One the contrary, another commonly-used flexure joint is a leaf spring or a compliant beam, the stroke of which is much larger due to its distributed-compliance characteristic. However, it lacks in precision and stiffness. In order to overcome such disadvantages, some complex flexure joints were inves- tigated recently (Tseytlin, 2006; Trease et al., 2005). The typical examples are the cross-axis flexural pivots (Jensen and Howell, 2002), the cartwheel hinges (Smith, 2000), the split-tube pivots (Goldfarb and Speich, 1999), the butterfly flexural pivot (Henein et al., 2003) and so on. Most of them consist of two and more flexural beams: a cross-axis flexural pivot is assembled by two beams; a cartwheel hinge is constructed by four beams; and butterfly pivot is up to eight beams.

Some complex flexure joints, such as the cross-axis flexural pivot, are indeed the spatial structures; therefore they are hard to be machined out from a monolithic block of material. Therefore, they are generally unsuitable for being used in the high precision systems because assembly error could be easily introduced.

A cartwheel hinge, on the contrary, has a planar nature. It provides not only a large-deflection stroke, but also overcomes such disadvantages of a cross-axis pivot as an unavoidable assembly and a relatively low rotational precision. As a result, by comparison with the cross-axis pivot, the cartwheel hinge gets a five times improvement in the stability of the center of rotation, but as a cost of nearly four times lose in the rotational stroke (Smith, 2000).

Correspondence to: X. Pei

(peixu@buaa.edu.cn) 
(a)

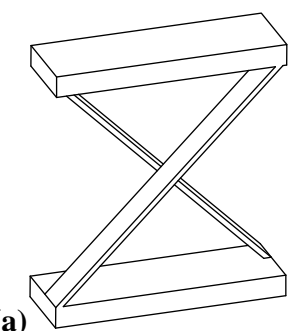

(b)

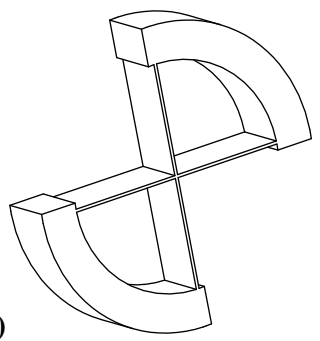

(c)

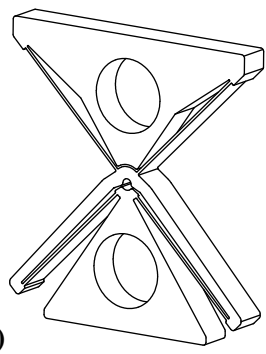

Figure 1. Leaf-Type Flexure joints: (a) a cross-axis pivot; (b) a cartwheel hinge; (c) an ADLIF.

Another type of planar flexure joint is the butterfly flexural pivot. Although there exists 8 beams in the architecture, it is a nearly perfect combination of a large-deflection stroke and a high precision (much better than a cartwheel hinge).

Whether for a cartwheel hinge or a butterfly flexural pivot, we notice that both of them can be regarded as the combination of two and more LITF pivots (Pei et al., 2008a), in which the remote-center-motion (RCM) enable them to be easily superimposed to construct some new complex flexure joints. Just inspired by this design phylosiphy, in this paper, we present a new type of a planar leaf-type flexure joint, i.e. an anti-symmetric double leaf-type isosceles-trapezoidal flexure joint (ADLIF), which is constructed by four beams. The ADLIF possesses almost all the merits of the cartwheel hinge and meantime can gain much higher precision. In the later sections, Finite Element Analysis (FEA) is used to validate such an improvement. In addition, in order to provide a simple tool to design or optimize the ADLIF, an improved pseudo-rigid-body (PRB) method is also formulated. The proposed PRB model is not only simple and accurate, but also intuitive to designers.

\section{Conceptual design}

As shown in Fig. 1c, an ADLIF consists of four leaf-type flexures, and the extended lines of the four leaves intersected at a point called the virtual pivot point. Both of the ADLIF and the cartwheel hinge can be regarded as two LITF building blocks connected in series (Pei et al., 2008a). The major difference between them is that whether the two LITF modules are arranged symmetrically or anti-symmetrically. In the ADLIF as shown in Fig. 2b, the two LITF modules are connected by an intermediate body. In a cartwheel hinge, the intermediate body is reduced to zero. When either of two rims (Rim 1 and Rim 2) is stationary, the other one becomes movable.

Three parameters are needed to determine the configuration of the ADLIF: (1) $h_{\mathrm{f}}$ denotes the distance between the bottom end of the leaves and the pivot point $O$; (2) $H$ denotes the distance between the upper end of the leaves and the pivot point $O$; (3) $\phi$ denotes half of the angle between two leaves. The length of each leaf is thus written as

$l=\overline{D A}=\left(H-h_{\mathrm{f}}\right) / \cos \phi$

Assuming one rim (Rim 1 or Rim 2) is stationary, when the joint deflects, $\theta$ is used to denote the rotation angle of the other rim.

\section{Pseudo-rigid-body model}

Although the FEA is an effective and credible way to analyze a flexible body, the commercial software is usually expensive; what is more important, the modelling and simulation processes are rather time-consuming. In the early design phases of flexures, a PRB is instead a useful tool. It is not only intuitive to designers, but also a resultant parameterized model that facilitates structural optimization. In this section, based on the results for the LITF model (Pei et al., 2008b, c), a simple PRB model of the ADLIF is formulated. The accuracy of the model is relatively high, and it can be confirmed in Sect. 5 by comparison with the results of FEA.

An improved PRB bar model of the ADLIF is shown in Fig. 2b, where the bold lines denote the rigid segments; eight ideal rigid pivots are added to predict the deflected path; four torsional springs are attached at the inner four pivots to reveal the force-deflection relationships in the ADLIF.

The vertical distances between all four pivots $A, B, A^{\prime}, B^{\prime}$ and the center $O$ are equal to $h$, which is given by

$h=\gamma h_{\mathrm{f}}+(1-\gamma) H$

where the characteristic radius factor $\gamma$ is defined using the equation (Pei et al., 2008b)

$\gamma=\frac{15}{2 n_{f}^{2}-n_{\mathrm{f}}+17}$.

$n_{\mathrm{f}}=h_{\mathrm{f}} / H$

Using a rough estimation, $\gamma$ is approximately equal to 8/9.

The stiffness of the spring $K$ can be calculated as (Pei et al., 2008b)

$K=\frac{4 E I \gamma^{2}\left(1+n_{\mathrm{f}}+n_{\mathrm{f}}^{2}\right)}{l_{\mathrm{f}}}$ 


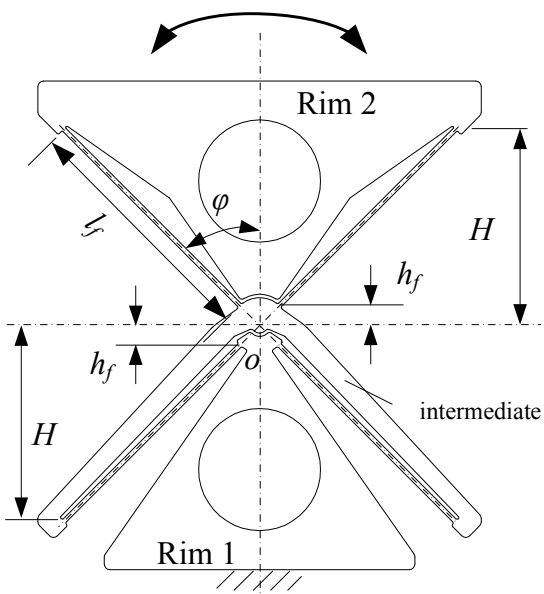

(a)

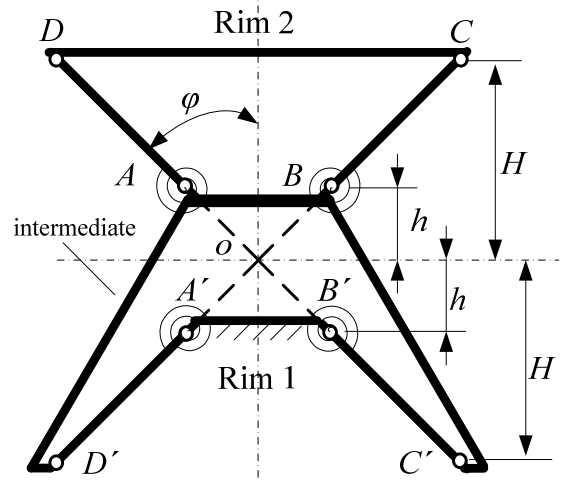

(b)

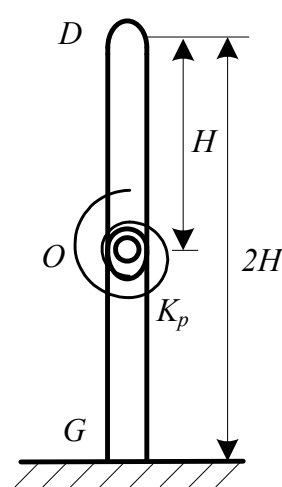

(c)

Figure 2. The PRB model of an ADLIF: (a) the ADLIF; (b) the bar model; (c) the pin-joint model.

\subsection{Stiffness}

With aid of the PRB bar model shown in Fig. 2b, the forcedisplacement relationship of the ADLIF can be found correspondingly. In addition, a pin-joint model of ADLIF the shown in Fig. $2 \mathrm{c}$ is proposed by simplifying the PRB bar model. The more detailed modeling process has been demonstrated extensively in the literature (Pei et al., 2008b). The stiffness value of the pin-joint model can be obtained by

$K_{\mathrm{p}}=\frac{4 E I\left(H^{2}+H h_{\mathrm{f}}+h_{\mathrm{f}}^{2}\right) \cos \phi}{\left(H-h_{\mathrm{f}}\right)^{3}}$

$M=K_{\mathrm{p}} \cdot \theta$

\subsection{Center-shift}

The developed pin-joint model only can be used to determine the moment-displacement nature. When the detail feature of the joint needs to be investigated, such as the accurate motion of the Rim 2, the bar model prefers to be used in this case. The center-shift $\delta$ is an important criterion for evaluating the rotational precision of a general flexure joint. It can be obtained in a way proposed in literature (Pei et al., 2008a). The vector-form result is given by

$\boldsymbol{\delta}=-\boldsymbol{\delta}_{1} \cos \frac{\theta}{2}+\boldsymbol{\delta}_{1} \sin \frac{\theta}{2} \cdot i$

$\|\boldsymbol{\delta}\|=\delta_{1} \sqrt{2\left(1-\cos \frac{\theta}{2}\right)}=\delta_{1}\left|\frac{\theta}{2}\right|$

where $\delta_{1}$ is the center-shift of a single LITF joint, and

$\delta_{1}=\delta_{1 \mathrm{x}}+i \delta_{1 \mathrm{y}}$

$\frac{\left\|\boldsymbol{\delta}_{1}\right\|}{H}=B_{3} \sqrt{B_{1}}$ where $\delta_{1 \mathrm{x}}$ and $\delta_{1 \mathrm{y}}$ are the components of the center-shift. They can be written as

$\frac{\delta_{1 \mathrm{x}}}{H}=B_{3} \cdot \sin \frac{\theta}{2}$

$\frac{\delta_{1 \mathrm{y}}}{H}=\left(n-\cos \frac{\theta}{2}\right) \cdot B_{3}$

where

$B_{1}=1+n^{2}-2 n \cos \frac{\theta}{2}$

$B_{2}=(1-n)^{2} / \sin ^{2} \varphi$

$B_{3}=1-\tan \varphi \sqrt{\frac{B_{2}}{B_{1}}-1}$

$n=h / H$

\subsection{Stress analysis}

Stress always occurs as the hinge deflects. It is therefore another important criterion to be considered, which can also be used to decide the maximum deflection for the hinge.

For a single flexible segment, because the length $l$ is much larger than the thickness $t$, thus the shear deflection can be neglected, and only the bending deflection is considered. In this case, the stress is given by

$\sigma_{\text {max }}=\frac{E t\left(2 H+h_{\mathrm{f}}\right) \cos \phi}{2\left(H-h_{\mathrm{f}}\right)^{2}} \theta$

The maximum deflection of the ADLIF may be found by replacing $\sigma$ with the yield strength $\left(S_{\mathrm{y}}\right)$.

$\theta_{\max }=\frac{2\left(H-h_{\mathrm{f}}\right)^{2}}{E t\left(2 H+h_{\mathrm{f}}\right) \cos \phi} S_{y}$ 


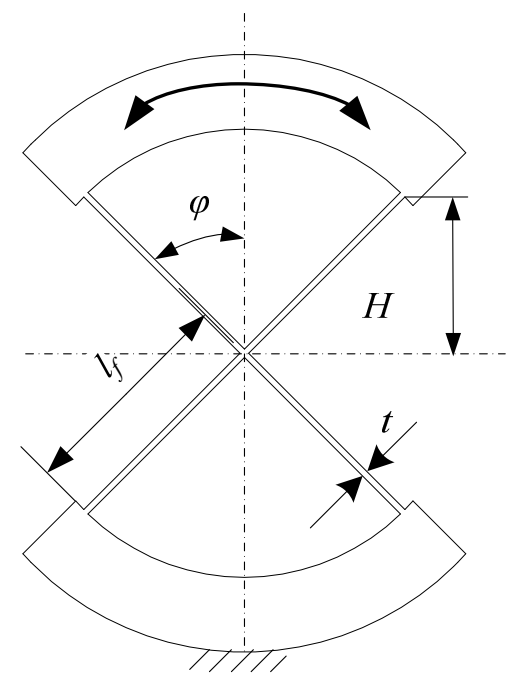

Figure 3. Parametric model of the cartwheel hinge.

\subsection{Model validation}

To validate the proposed PRB model, two ADLIFs with different structural parameters are adopted in thus study, and the results are plotted in Fig. 4 to compare with FEA simulation results. Figure 4 shows that the curves of the PRB and FEA results are very close to each other.

\section{Case study}

In order to validate the improvement in performances, two ADLIFs with different structural parameters are selected to make a comparison with a cartwheel hinge. Figure 3 illustrates the parametric model for the cartwheel hinge, and all structural parameters in the ADLIF are described in Fig. 2a. The concrete parameter values used for comparison are listed in Table 1. The length of leaves of the first ADLIF (ADLIF I) is same as that of the cartwheel joint, while the stiffness specification of the second ADLIF (ADLIF II) is approximate to that of the cartwheel joint. In Table $1, t$ is the thickness of the flexible segment; $b$ is the width of the flexible segment; $E$ is the Young's modulus.

The commercial FEA software (ANSYS 9.0) capable of making a large-deflection nonlinear analysis is used. The selected material in these cases is aluminum alloy. The Young's modulus, $E$, is thus $71 \mathrm{GPa}$ and the Poisson's ratio, $\mu$ is 0.33 . The BEAM3 elements have been chosen with the large displacement option turned on. The options of non-linear computation and stress stiffening are also turned on. A moment is loaded in the middle of moveable rim for each joint; while the other rim is fixed on the ground. (a)

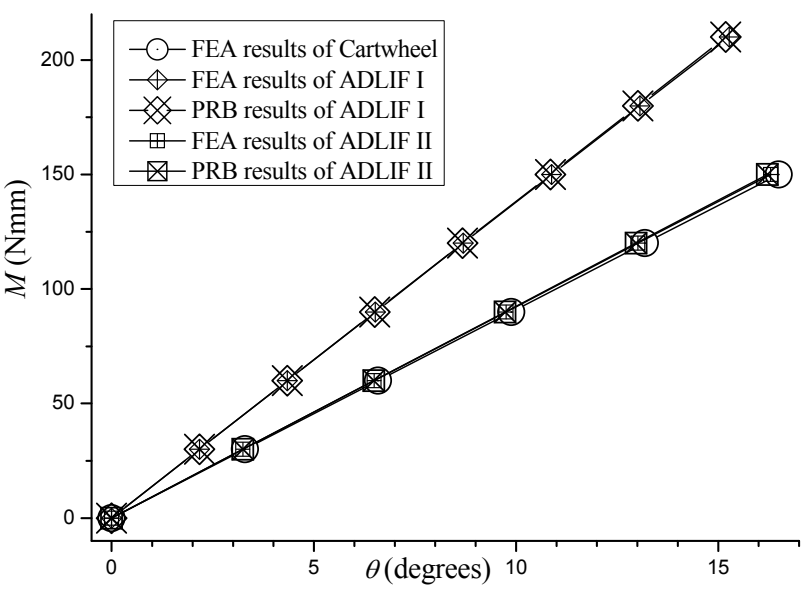

(b)

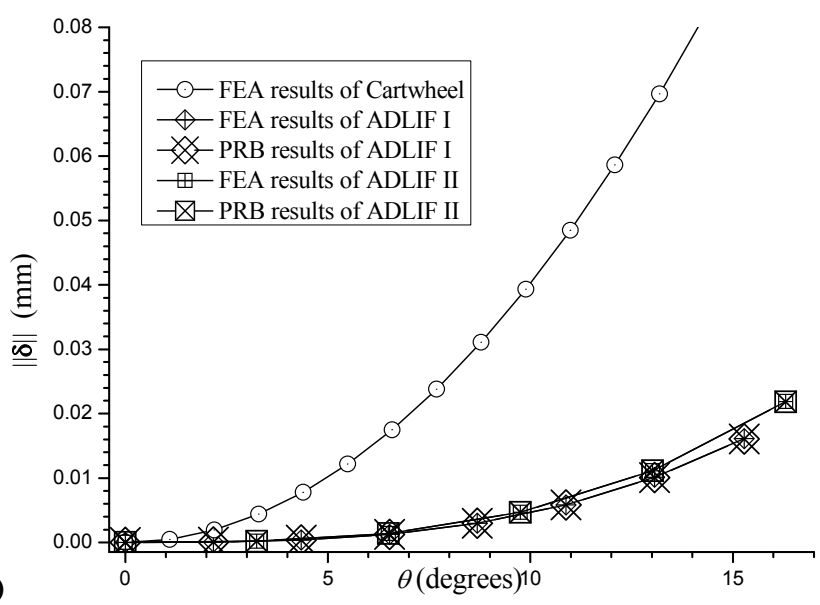

(c)

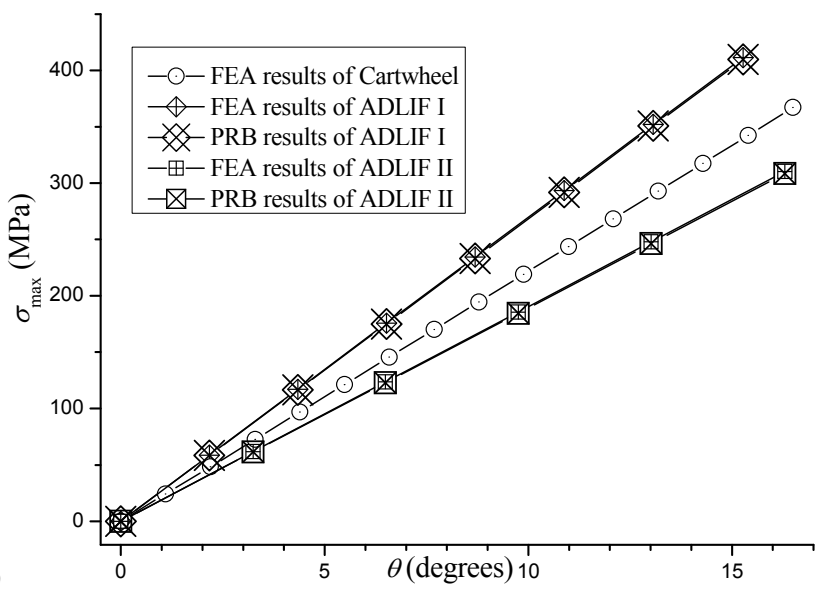

Figure 4. Performance comparisons of a cartwheel hinge and two ADLIFs: (a) Stiffness; (b) Center-shift; (c) Stress. 
Table 1. Structural parameters of three flexure joints.

\begin{tabular}{lccccccc}
\hline & $H(\mathrm{~mm})$ & $h_{\mathrm{f}}(\mathrm{mm})$ & $\phi($ degree $)$ & $l_{\mathrm{f}}(\mathrm{mm})$ & $t(\mathrm{~mm})$ & $b(\mathrm{~mm})$ & $E(\mathrm{Mpa})$ \\
\hline Cartwheel & 20 & - & 45 & 28.28 & 0.5 & 5 & 71000 \\
ADLIF I & 23 & 3 & 45 & 28.28 & 0.5 & 5 & 71000 \\
ADLIF II & 30 & 3 & 45 & 38.18 & 0.5 & 5 & 71000 \\
\hline
\end{tabular}

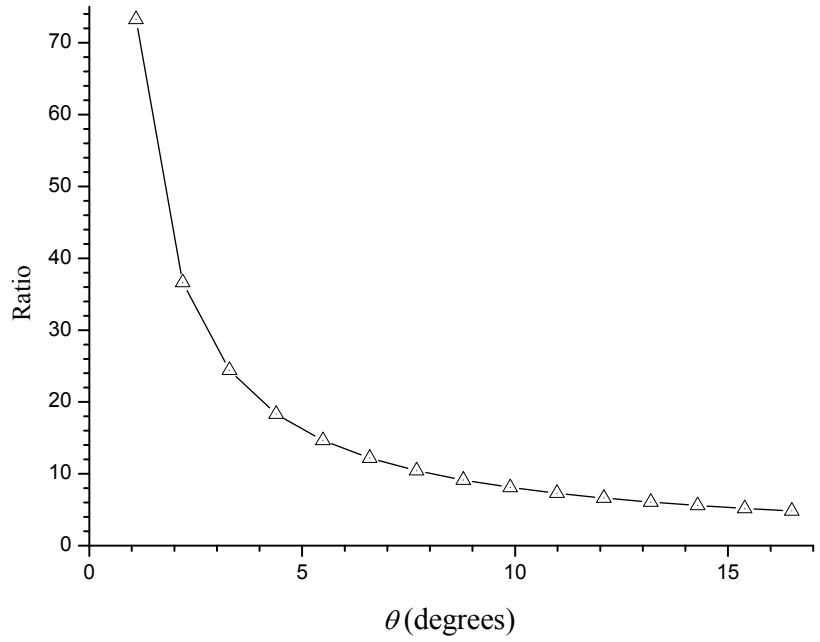

Figure 5. Center-shifts ratio of the cartwheel hinge to the ADLIF II.

\section{Results and discussion}

The moment-displacement characteristics, center-shifts, and stresses of the three flexure joints are measured and evaluated through the FEA program, and the results are ploted in Fig. 4.

$h_{\mathrm{f}}$ of ADLIF cannot be too small due to the limit of the manufacture capability. This makes the stiffness value of the ADLIF I higher than the cartwheel hinge (shown in Fig. 4a). When the length of all leaves is identical to each other, increase in the leaves' length of the ADLIF (see ADLIF II) may lead to the reduced stiffness. Meanwhile, the center-shift of the ADLIF II increases by a little, as shown in Fig. 4b, and it is still much smaller than that of the cartwheel hinge.

As illustrated in Fig. 5, the center-shift ratio of the cartwheel hinge to the ADLIF II is decreasing when the rotational angle becomes larger. When the rotational angle is up to $5^{\circ}$, the center-shift of the cartwheel joint (about $0.01 \mathrm{~mm}$ ) is about 16 times larger than that of ADLIF II (about $0.0006 \mathrm{~mm}$ ). In other words, by comparison with the cartwheel hinge, the ADLIF II can gain more than 16 times improvement in precision when rotational angle is less than $5^{\circ}$.

The maximum deflections of all three joints can be found in Fig. 4c. If the yield strength $S_{\mathrm{y}}$ is $250 \mathrm{MPa}$, the ranges of motion corresponding to the cartwheel hinge, the ADLIF I and the ADLIF II are about $11.4^{\circ}, 9.3^{\circ}$ and $13.2^{\circ}$, respectively.

\section{Conclusions}

A novel large-displacement flexure joint, named an ADLIF, is proposed in this paper. It consists of two LITF building blocks, but these two blocks are arranged asymmetrically. Compared with the cartwheel hinge commonly used in precision engineering and characterized as two LITF building blocks arranged symmetrically, the ADLIF can gain a great improvement in precision as well as keeping other characteristics such as stiffness and ranges of motion at the same level. In order to quantitatively evaluate these performances, A PRB model of the ADLIF is developed, and the moment-displacement characteristics, center-shifts, and stresses of two cases are calculated correspondingly. By aid of validation by the FEA result, the PRB model is proved accurate. According to both the theoretical and simulation results, the ADLIF can get more than 16 times improvement in precision as the rotational angle is less than $5^{\circ}$. Therefore, the ADLIF is suitable for a replacement of the cartwheel hinge in precision some applications.

Acknowledgements. The authors would like to acknowledge the support of National Natural Science Foundation of China, through Grant No. 50905005, 50875008.

Edited by: N. Tolou

Reviewed by: G. Chen, A. Khavvaji, and

another anonymous referee

\section{References}

Goldfarb, M. and Speich, J.: A Well-Behaved Revolute Flexure Joint for Compliant Mechanism Design, ASME J. Mech. Des., 121, 424-429, 1999.

Henein, S., Droz, S., Myklebust, L., and Onillon, E.: Flexure pivot for aerospace mechanisms, Proc. 10th European Space Mechanisms and Tribology Symposium, 24-26 September 2003, San Sebastian, Spain, 1-4, 2003.

Her, I. and Chang, J. C.: A linear scheme for the displacement analysis of micropositioning stages with flexure hinges, J. Mech. Design, 116, 770-776, 1994.

Jensen, B. D. and Howell, L. L.: The modeling of cross-axis flexural pivots, Mech. Mach. Theory, 37, 461-476, 2002.

Kota, S., Hetrick, J., Li, Z., and Saggere, L.: Tailoring unconventional actuators using compliant transmissions: design methods and applications, IEEE/ASME Transactions on Mechatronics, 4, 396-408, 1999. 
Lobontiu, N.: Compliant Mechanisms: Design of Flexure Hinges, CRC Press, Boca Raton, FL, 2003.

Onillon, E., Henein, S., and Theurillat, P.: Small scanning mirror mechanism, 2003 IEEE/ASME International Conference on Advanced Intelligent Mechatronics, 1129-1133, 2003.

Pei, X., Yu, J. J., Zong, G. H., and Bi, S. S.: A Novel Family of Leaf-Type Compliant Joints: Combination of Two IsoscelesTrapezoidal Flexural Pivots in Series, Journal of Mechanisms and Robotics, 1, 021005, 1-6, 2008a.

Pei, X., Yu, J. J., Zong, G. H., and Bi, S. S.: The stiffness model of leaf-type isosceles-trapezoidal flexural pivots, J. Mech. Design, 130, 082303, doi:10.1115/1.2936902, 2008b.

Pei, X., Yu, J. J., Zong, G. H., Bi, S. S., and Yu, Z. W.: Analysis of rotational precision for an isosceles-trapezoidal flexural pivot, J. Mech. Design, 130, 052302 doi:10.1115/1.2885507, 2008c.
Pernette, E., Henein, S., Magnani, I., and Clavel, R.: Design of parallel robots in microrobotics, Robotica, 15, 417-420, 1997.

Slocum, A. H.: Precision Machine Design, Society of Manufacturing Engineers, 1992.

Smith, S. T.: Flexures: elements of elastic mechanisms, Gordon and Breach Science, New York, 153-230, 2000.

Trease, B. P., Moon, Y. M., and Kota, S.: Design of LargeDisplacement Compliant Joints, J. Mech. Design, 127, 788-798, 2005.

Tseytlin, Y. M.: Structural Synthesis in Precision Elasticity, Springer, New York, 2006. 